for

\title{
HEADSPACE PASSIVE DOSING OF VOLATILE HYDROPHOBIC ORGANIC CHEMICALS FROM A LIPID DONOR - LINKING THEIR TOXICITY TO WELL-DEFINED EXPOSURE FOR AN IMPROVED RISK ASSESSMENT
}

\author{
Lam Ngoc Trac ${ }^{1}$, Stine Nørgaard Schmidt ${ }^{1}$, Martin Holmstrup ${ }^{2}$, Philipp Mayer ${ }^{* 1}$ \\ ${ }^{1}$ Department of Environmental Engineering, Technical University of Denmark, DK-2800 Kgs. Lyngby, \\ Denmark
}

${ }^{2}$ Department of Bioscience, Aarhus University, DK-8600 Silkeborg, Denmark

*Phone: +45 4525 1569; fax: +45 4593 2850; e-mail: philm@env.dtu.dk

Current affiliation for Stine Nørgaard Schmidt: National Research Council, U.S. Environmental Protection Agency, ORD/NHEERL Atlantic Ecology Division, Narragansett, Rhode Island, USA.

Number of pages: 9

Number of figures: 4

Number of tables: 4

This Supporting Information contains the following:

- Linearity check for the chemical activity measurements (Figure S1).

- Algal growth in control samples in the algal growth inhibition tests (Figure S2).

- Toxicity of other test chemicals in the algal growth inhibition and springtail lethality tests on the lipid donor basis (Figure S3) and the membrane basis (Figure S4).

- Median effective concentrations (EC-50s) in lipid donor and lipid membrane of all test chemicals (Table S1).

- Median effective chemical activities (Ea-50s) of limonene, n-nonane, octamethylcyclotetrasiloxane (D4), and decamethylcyclopentasiloxane (D5) (Table S2).

- Data for the chemical activity measurements of limonene, n-nonane, and octamethylcyclotetrasiloxane (D4) (Table S3).

- Dose-response data on the lipid donor basis for algal growth inhibition and springtail lethality tests (Table S4). 

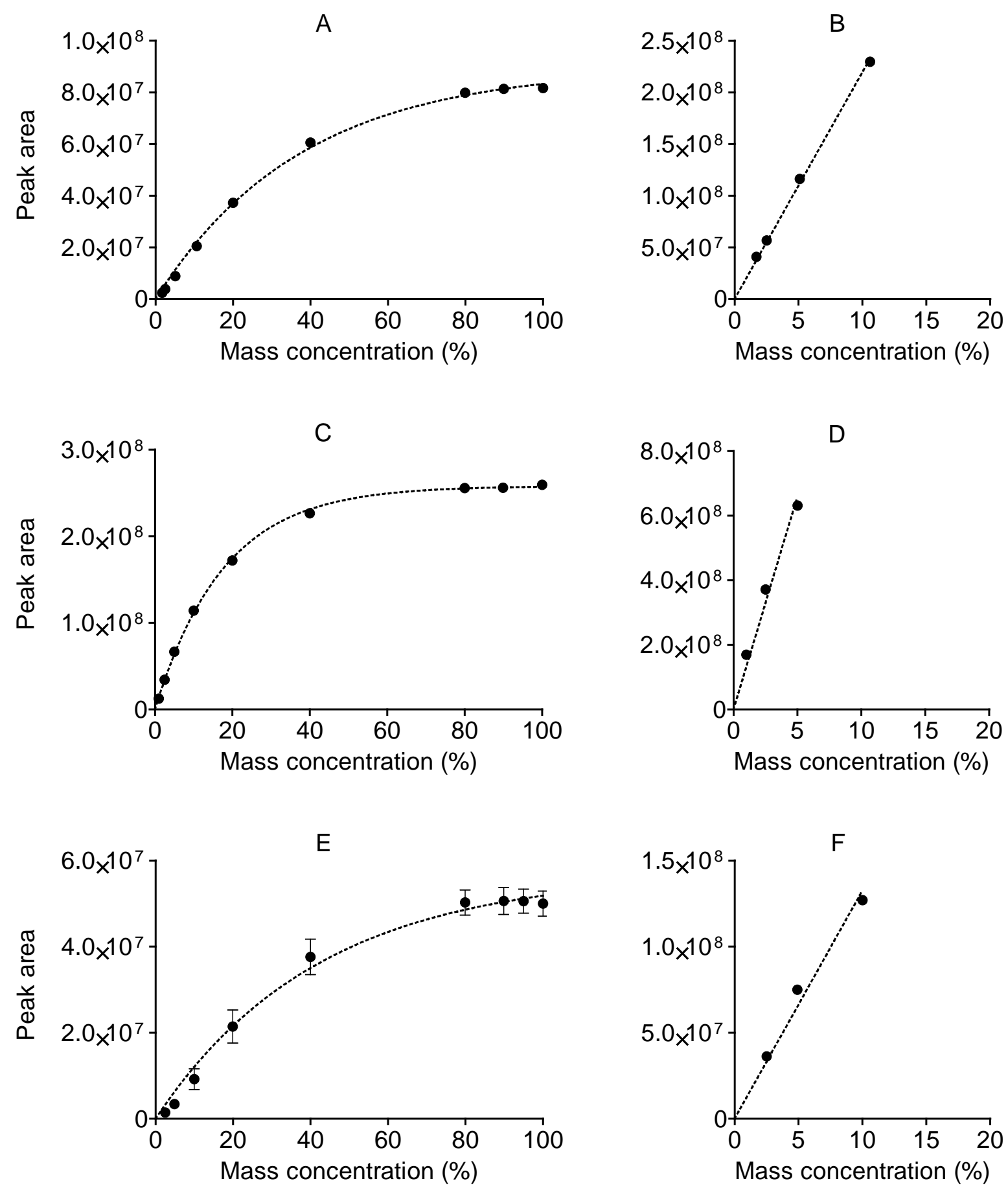

Figure S1: Headspace GC-MS measurements with a split ratio of 100:1 (mean $\pm S E M, n=3$ ) and subsequent linearity checks with a split ratio of 10:1 (mean \pm SEM, $n=2$ ) for limonene $(A) \&(B)$, n-nonane $(C) \&(D)$, and octamethylcyclotetrasiloxane D4 (E) \& (F), respectively. Deviation from linearity tests by GraphPad Prism version 5.0 (GraphPad Software, Inc., USA) for (B), (D) \& (F) were not significant. 

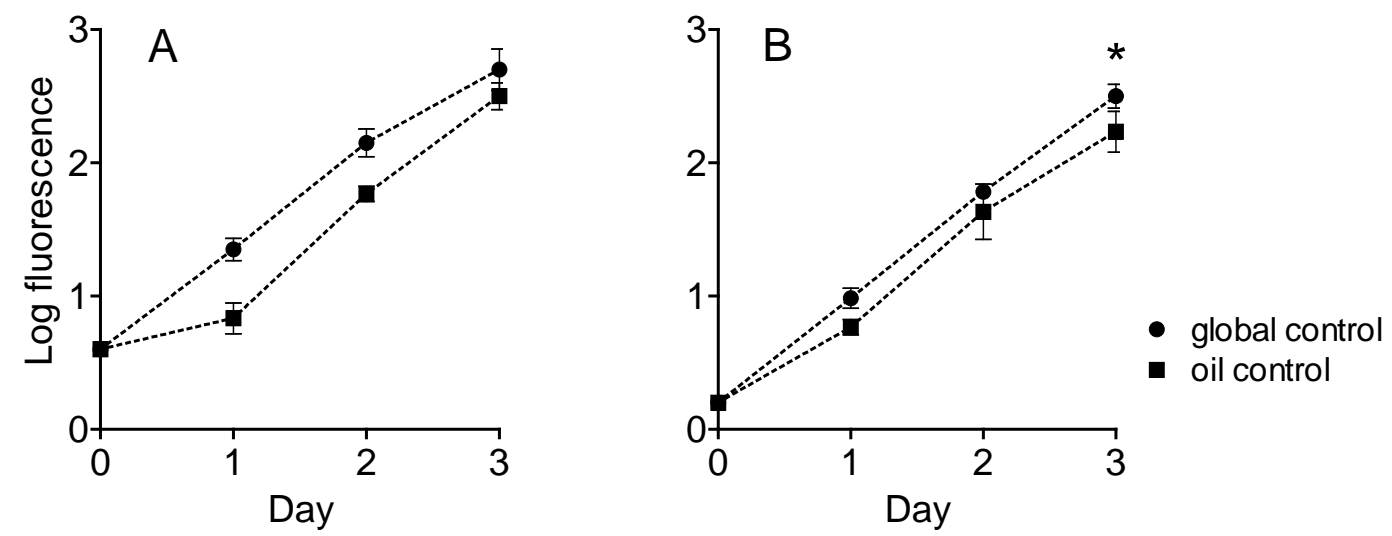

Figure S2: Algal biomass measured for the global control (mean $\pm S D, n=6$ ) and the oil control (mean $\pm S D$, $n=3$ ) throughout the tests with terpenes $(A)$ and alkanes $(B)$. The asterisk shows a minor but statistically significant difference $(p<0.05, t$-test) between the global and oil controls on day 3 .

\section{R. subcapitata}
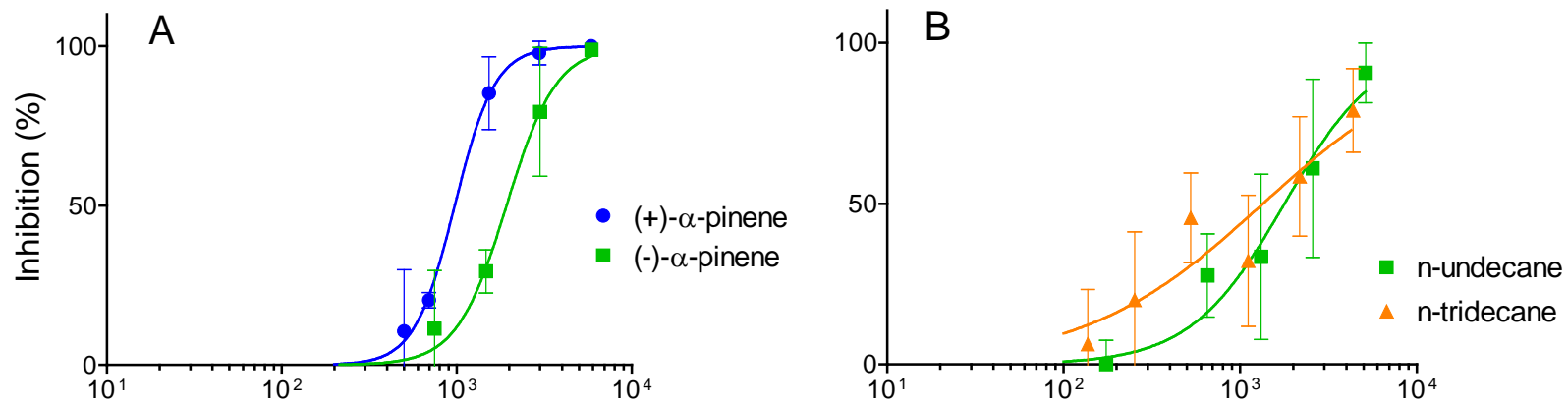

F. candida
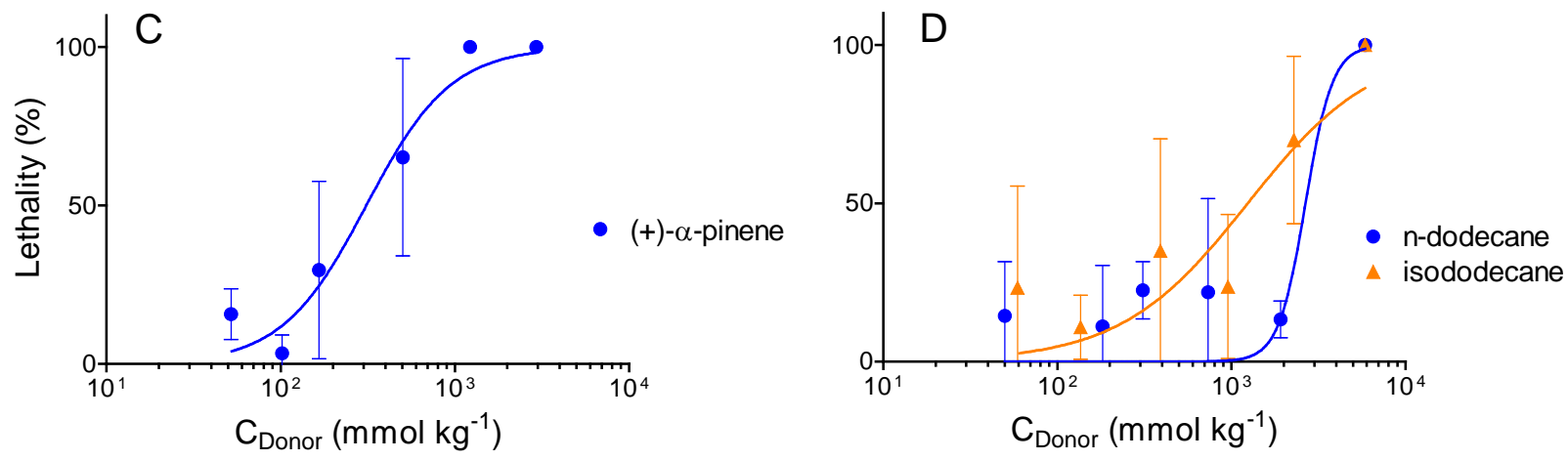

Figure S3: Toxicity of terpenes and alkanes to algae (A \& B) and springtail (C \& D) assessed by concentration in lipid donor (mean $\pm S D, n=3$ ). 


\section{R. subcapitata}
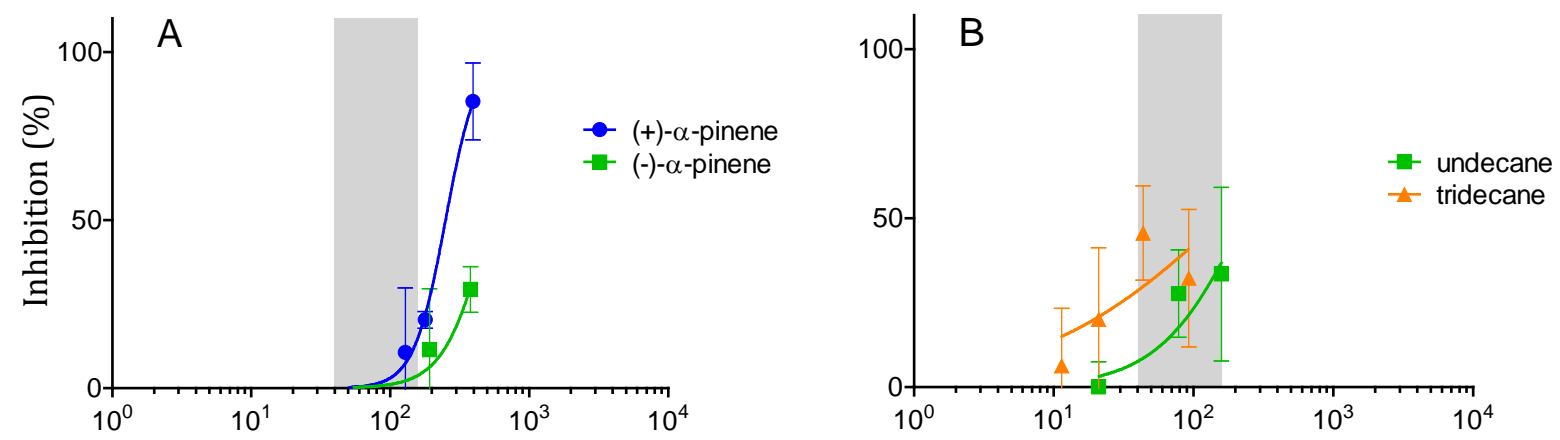

$\mathrm{C}_{\text {Membrane }} \rightleftharpoons$ Donor $\left(\mathrm{mmol} \mathrm{kg}^{-1}\right)$

$\mathrm{C}_{\text {Membrane }} \rightleftharpoons$ Donor $\left(\mathrm{mmol} \mathrm{kg}^{-1}\right)$

\section{F. candida}
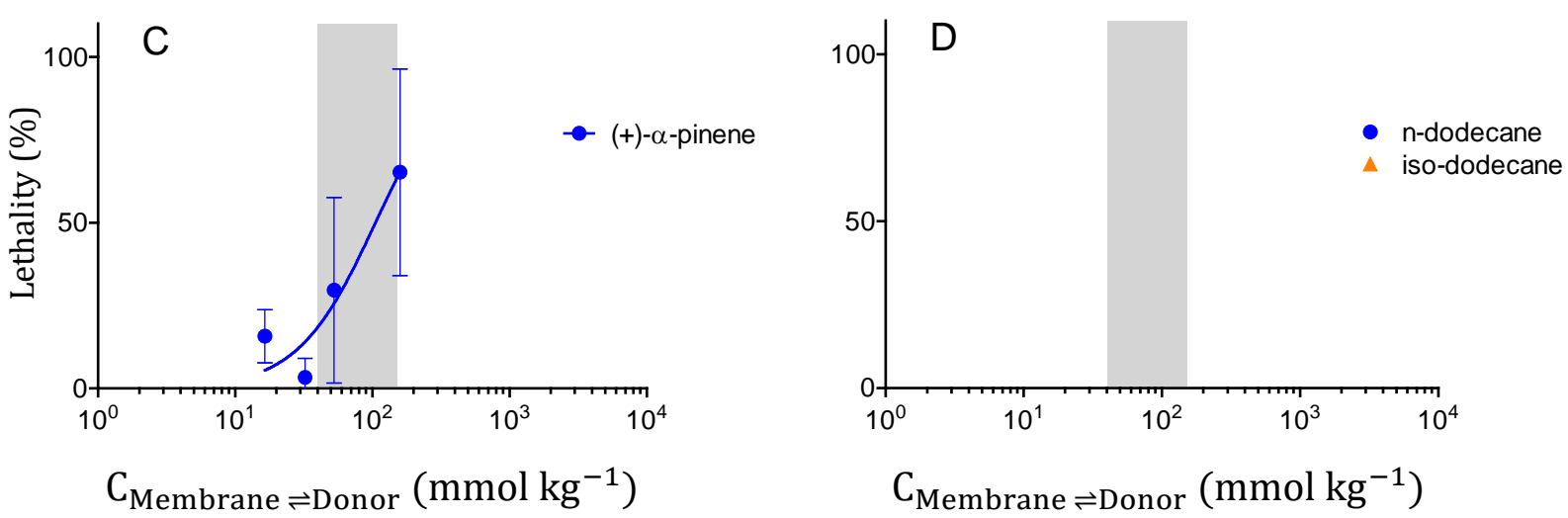

Figure S4: Toxicity of terpenes and alkanes to algae (A \& B) and springtail (C \& D) assessed by concentration in membranes at equilibrium with lipid donor (mean $\pm S D, n=3$ ), assuming a linear partitioning between storage lipid and membrane lipid up to $\mathrm{C}_{\text {Donor }}=30 \%$ for these chemicals. For test chemicals without clear toxicity at $\mathrm{C}_{\mathrm{Donor}}=30 \%$, no dose-response curves were thus produced. The grey box depicts the concentration range of $40-160 \mathrm{mmol} \mathrm{kg}^{-1}$ lipid for baseline toxicity. 
Table S1: Median effective concentrations (EC-50s) in lipid donor and lipid membrane of all test chemicals in algal growth inhibition tests and springtail lethality tests. The values are shown as Mean $(95 \%$ confidence interval; $r^{2}$ value). L.T.: limited toxicity (11\% growth inhibition at the saturation level), N.T.: no toxicity at saturation level, N.I.: not included, N.D.: not derivable due to no toxicity up to mass concentration of $30 \%$ (see Section 2.4.2).

\begin{tabular}{|c|c|c|c|c|}
\hline & \multicolumn{2}{|c|}{ Algal growth inhibition tests } & \multicolumn{2}{|c|}{ Springtail lethality tests } \\
\hline & $\begin{array}{c}\text { Donor EC-50 } \\
\text { (mmol kg } \mathrm{mm}^{-1} \text { lipid) }\end{array}$ & $\begin{array}{l}\text { Membrane EC-50 } \\
\text { (mmol kg-1 lipid) }\end{array}$ & $\begin{array}{c}\text { Donor EC-50 } \\
\text { (mmol kg } \mathrm{mg}^{-1} \text { lipid) }\end{array}$ & $\begin{array}{l}\text { Membrane EC-50 } \\
\text { (mmol kg }{ }^{-1} \text { lipid) }\end{array}$ \\
\hline n-nonane & $\begin{array}{c}730 \\
\left(620-870 ; r^{2}=0.9257\right)\end{array}$ & $\begin{array}{c}130 \\
\left(100-160 ; r^{2}=0.8533\right)\end{array}$ & $\begin{array}{c}500 \\
\left(420-600 ; r^{2}=0.8878\right)\end{array}$ & $\begin{array}{c}90 \\
\left(70-110 ; r^{2}=0.8400\right)\end{array}$ \\
\hline n-undecane & $\begin{array}{c}1780 \\
\left(1280-2490 ; r^{2}=0.7997\right)\end{array}$ & $\begin{array}{c}230 \\
\left(90-590 ; r^{2}=0.4680\right)\end{array}$ & N.I. & N.I. \\
\hline n-dodecane & N.I. & N.I. & $\begin{array}{c}2670 \\
\left(800-8860 ; r^{2}=0.6547\right)\end{array}$ & N.D. \\
\hline n-tridecane & $\begin{array}{c}1350 \\
\left(790-2300 ; r^{2}=0.6366\right)\end{array}$ & $\begin{array}{c}160 \\
\left(20-1320 ; r^{2}=0.2547\right)\end{array}$ & N.I. & N.I. \\
\hline isododecane & N.I. & N.I. & $\begin{array}{c}1240 \\
\left(630-2460 ; r^{2}=0.6000\right)\end{array}$ & N.D. \\
\hline Limonene & $\begin{array}{c}690 \\
(95 \% \mathrm{Cl} \text { could not be } \\
\left.\text { calculated; } r^{2}=0.9845\right)\end{array}$ & $\begin{array}{c}210 \\
(95 \% \mathrm{Cl} \text { could not be } \\
\left.\text { calculated; } \mathrm{r}^{2}=0.9778\right)\end{array}$ & $\begin{array}{c}290 \\
\left(55-1500 ; r^{2}=0.9787\right)\end{array}$ & $\begin{array}{c}90 \\
\left(10-800 ; r^{2}=0.9578\right)\end{array}$ \\
\hline$(+)-\alpha$-pinene & $\begin{array}{c}970 \\
\left(850-1110 ; r^{2}=0.9639\right)\end{array}$ & $\begin{array}{c}250 \\
\left(210-300 ; r^{2}=0.9187\right)\end{array}$ & $\begin{array}{c}310 \\
\left(210-450 ; r^{2}=0.8465\right)\end{array}$ & $\begin{array}{c}90 \\
\left(50-150 ; r^{2}=0.5652\right)\end{array}$ \\
\hline (-)-a-pinene & $\begin{array}{c}1930 \\
\left(1600-2300 ; r^{2}=0.9270\right)\end{array}$ & $\begin{array}{c}510 \\
\left(290-930 ; r^{2}=0.6073\right)\end{array}$ & N.I. & N.I. \\
\hline D4 & L.T.\# & L.T.\# & $\begin{array}{c}680 \\
\left(470-960 ; r^{2}=0.8797\right)\end{array}$ & $\begin{array}{c}70 \\
\left(50-110 ; r^{2}=0.7678\right)\end{array}$ \\
\hline D5 & N.T.\# & N.T. ${ }^{\#}$ & $\begin{array}{c}1670 \\
\left(870-3170 ; r^{2}=0.6709\right)\end{array}$ & $\begin{array}{c}70 \\
\left(50-90 ; r^{2}=0.7448\right)\end{array}$ \\
\hline
\end{tabular}

\footnotetext{
\#: data from Trac et al. (Trac et al., 2018).
} 
Table S2: Median effective chemical activities (Ea-50s) of limonene, n-nonane, octamethylcyclotetrasiloxane (D4), and decamethylcyclopentasiloxane (D5) in algal growth inhibition tests and springtail lethality tests. The values are shown as Mean ( $95 \%$ confidence interval; $r^{2}$ value). L.T.: limited toxicity ( $11 \%$ growth inhibition at the saturation level), N.T.: no toxicity at saturation level, N.D.: not determined.

\begin{tabular}{|c|c|c|}
\hline & Algal growth inhibition tests & Springtail lethality tests \\
\hline & $\begin{array}{c}\text { Ea-50 } \\
\text { (unitless) }\end{array}$ & $\begin{array}{c}\text { Ea-50 } \\
\text { (unitless) }\end{array}$ \\
\hline Limonene & $\begin{array}{c}0.25 \\
\left(95 \% \mathrm{Cl} \text { could not be calculated; } r^{2}=0.9845 \text { ) }\right.\end{array}$ & $\begin{array}{c}0.11 \\
\left(0.05-0.24 ; r^{2}=0.9787\right)\end{array}$ \\
\hline n-nonane & $\begin{array}{c}0.40 \\
\left(0.35-0.45 ; r^{2}=0.9099\right)\end{array}$ & $\begin{array}{c}0.30 \\
\left(0.25-0.35 ; r^{2}=0.8873\right)\end{array}$ \\
\hline D4 & L.T. ${ }^{\#}$ & $\begin{array}{c}0.42 \\
\left(0.31-0.56 ; r^{2}=0.8782\right)\end{array}$ \\
\hline D5 & N.T.\# & N.D. \\
\hline
\end{tabular}

\#: data from Trac et al. (Trac et al., 2018). 
Table S3: Data for the chemical activity measurements of limonene, n-nonane, and octamethylcyclotetrasiloxane (D4).

\begin{tabular}{|c|c|c|c|c|c|c|c|}
\hline \multirow{2}{*}{$\begin{array}{c}\mathrm{C}_{\text {Donor }} \\
\text { (mass \%) }\end{array}$} & \multicolumn{3}{|c|}{ Chemical activity } & \multirow{2}{*}{$\begin{array}{c}\mathrm{C}_{\text {Donor }} \\
\text { (mass \%) }\end{array}$} & \multicolumn{3}{|c|}{ Chemical activity } \\
\hline & Rep 1 & Rep 2 & Rep 3 & & Rep 1 & Rep 2 & Rep 3 \\
\hline \multicolumn{5}{|c|}{ Limonene } & \multicolumn{3}{|c|}{ D4 } \\
\hline 0.0 & 0.00 & 0.00 & 0.00 & 0.0 & 0.00 & 0.00 & 0.00 \\
\hline 1.7 & 0.03 & 0.03 & 0.03 & 2.5 & 0.04 & 0.02 & 0.02 \\
\hline 2.5 & 0.06 & 0.04 & 0.04 & 4.9 & 0.09 & 0.06 & 0.05 \\
\hline 5.1 & 0.12 & 0.10 & 0.11 & 10.0 & 0.25 & 0.16 & 0.13 \\
\hline 10.6 & 0.26 & 0.24 & 0.25 & 19.9 & 0.51 & 0.42 & 0.33 \\
\hline 20.0 & 0.45 & 0.44 & 0.48 & 40.0 & 0.81 & 0.76 & 0.68 \\
\hline 40.0 & 0.75 & 0.73 & 0.75 & 79.9 & 1.01 & 0.99 & 1.02 \\
\hline 79.9 & 0.95 & 0.97 & 1.01 & 89.9 & 1.01 & 1.03 & 1.00 \\
\hline 89.9 & 0.97 & 0.98 & 1.03 & 95.0 & 1.00 & 1.03 & 1.01 \\
\hline \multirow[t]{2}{*}{100.0} & 1.00 & 1.00 & 1.00 & 100.0 & 1.00 & 1.00 & 1.00 \\
\hline & \multicolumn{3}{|c|}{ n-nonane } & & & & \\
\hline 0.0 & 0.00 & 0.00 & 0.00 & & & & \\
\hline 1.0 & 0.05 & 0.05 & 0.05 & & & & \\
\hline 2.5 & 0.14 & 0.13 & 0.13 & & & & \\
\hline 5.0 & 0.26 & 0.25 & 0.25 & & & & \\
\hline 10.0 & 0.45 & 0.44 & 0.43 & & & & \\
\hline 20.0 & 0.67 & 0.65 & 0.67 & & & & \\
\hline 40.0 & 0.89 & 0.86 & $1.19^{*}$ & & & & \\
\hline 80.0 & 0.99 & 0.98 & 0.99 & & & & \\
\hline 89.9 & 1.01 & $1.73^{*}$ & 0.98 & & & & \\
\hline 100.0 & 1.00 & 1.00 & 1.00 & & & & \\
\hline
\end{tabular}

* excluded for analysis as outliner, because peak areas deviated more than 3 times the standard deviation from the average of the other two replicates and were higher than the maximum chemical activity $\left(a_{\max }=1\right)$. 
Table S4: Dose-response data on a lipid donor basis for algal growth inhibition and springtail lethality tests. N. A.: not available due to invalid test vial.

\begin{tabular}{|c|c|c|c|c|c|c|c|c|c|}
\hline \multicolumn{5}{|c|}{ Algal growth inhibtion } & \multicolumn{5}{|c|}{ Springtail lethality } \\
\hline $\begin{array}{c}C_{\text {Donor }} \\
\text { (mass \%) }\end{array}$ & $\begin{array}{c}\text { CDonor } \\
(\mathrm{mmol} / \mathrm{kg})\end{array}$ & Rep 1 & Rep 2 & $\operatorname{Rep} 3$ & $\begin{array}{c}C_{\text {Donor }} \\
\text { (mass \%) }\end{array}$ & $\begin{array}{c}\left.\text { CDonor }_{\text {Dol }} \mathrm{kg}\right) \\
(\mathrm{mmol}\end{array}$ & Rep 1 & Rep 2 & Rep 3 \\
\hline \multicolumn{7}{|c|}{ Limonene } & \multicolumn{3}{|c|}{ Limonene } \\
\hline 79.6 & 5846 & 100.0 & 100.0 & 100.0 & 40.4 & 2967 & 100.0 & 100.0 & 100.0 \\
\hline 40.1 & 2946 & 100.0 & 100.0 & 100.0 & 15.7 & 1154 & 100.0 & 100.0 & 100.0 \\
\hline 20.6 & 1514 & 100.0 & 100.0 & 100.0 & 6.8 & 502 & 100.0 & 100.0 & 100.0 \\
\hline 10.0 & 735 & 75.7 & 77.3 & 100.0 & 3.0 & 218 & 0.0 & 0.0 & 11.1 \\
\hline 5.4 & 398 & -8.4 & -5.6 & 5.2 & 1.6 & 118 & 12.5 & 20.0 & 0.0 \\
\hline 3.1 & 224 & -5.5 & -8.1 & -7.4 & 0.4 & 28 & 10.0 & 10.0 & 0.0 \\
\hline \multicolumn{7}{|c|}{$(+)$-a-pinene } & \multicolumn{3}{|c|}{ (+)-a-pinene } \\
\hline 79.8 & 5861 & 100.0 & 100.0 & 100.0 & 40.0 & 2935 & 100.0 & 100.0 & 100.0 \\
\hline 40.3 & 2960 & 100.0 & 100.0 & 93.6 & 16.7 & 1222 & 100.0 & 100.0 & 100.0 \\
\hline 20.9 & 1533 & 78.6 & 78.8 & 98.5 & 6.9 & 503 & 55.6 & 40.0 & 100.0 \\
\hline 9.5 & 695 & 21.6 & 21.9 & 17.5 & 2.3 & 166 & 33.3 & 0.0 & 55.6 \\
\hline 6.8 & 500 & 32.0 & -5.3 & 5.2 & 1.4 & 102 & 10.0 & 0.0 & 0.0 \\
\hline 2.7 & 199 & -11.8 & -7.6 & -5.7 & 0.7 & 52 & 11.1 & 11.1 & 25.0 \\
\hline \multicolumn{10}{|c|}{ (-)-a-pinene } \\
\hline 80.3 & 5893 & 96.7 & 100.0 & 100.0 & & & & & \\
\hline 40.9 & 3000 & 59.5 & 78.8 & 100.0 & & & & & \\
\hline 20.1 & 1472 & 36.0 & 22.4 & 29.7 & & & & & \\
\hline 10.2 & 747 & 32.3 & 2.8 & -0.9 & & & & & \\
\hline 5.5 & 405 & -7.2 & -16.2 & -11.4 & & & & & \\
\hline 2.9 & 211 & -7.2 & -12.4 & -7.0 & & & & & \\
\hline \multicolumn{7}{|c|}{ n-nonane } & \multicolumn{3}{|c|}{ n-nonane } \\
\hline 79.9 & 6233 & 100.0 & 100.0 & 100.0 & 100.0 & 7797 & 100.0 & 100.0 & 100.0 \\
\hline 40.4 & 3149 & 100.0 & 100.0 & 100.0 & 79.4 & 6189 & 100.0 & 100.0 & 100.0 \\
\hline 20.4 & 1591 & 98.3 & 78.4 & 99.9 & 39.9 & 3107 & 100.0 & 100.0 & 100.0 \\
\hline 9.7 & 757 & 36.6 & 48.1 & 52.3 & 19.9 & 1549 & 100.0 & 100.0 & 100.0 \\
\hline 5.3 & 413 & 14.5 & 35.5 & 18.6 & 9.7 & 759 & 100.0 & 100.0 & 90.0 \\
\hline \multirow[t]{2}{*}{3.6} & 284 & 35.1 & 13.2 & -7.2 & 5.3 & 410 & 33.3 & 0.0 & 20.0 \\
\hline & & & & & 3.0 & 233 & 0.0 & 25.0 & 44.4 \\
\hline \multicolumn{7}{|c|}{ n-undecane } & \multicolumn{3}{|c|}{ n-dodecane } \\
\hline 80.1 & 5125 & 90.6 & 100.0 & 81.4 & 100.0 & 5872 & 100.0 & 100.0 & 100.0 \\
\hline 40.3 & 2581 & 42.6 & 19.7 & 20.8 & 32.6 & 1917 & 20.0 & 10.0 & 10.0 \\
\hline 20.6 & 1315 & 29.0 & 75.5 & 78.4 & 12.5 & 736 & 0.0 & 10.0 & 55.6 \\
\hline 10.2 & 651 & 8.5 & -5.3 & -2.8 & 5.3 & 310 & 30.0 & 25.0 & 12.5 \\
\hline 5.0 & 321 & 8.5 & 32.1 & 59.8 & 3.1 & 182 & 0.0 & 33.3 & 0.0 \\
\hline 2.7 & 174 & -11.5 & 13.9 & -6.6 & 0.8 & 50 & 10.0 & 33.3 & 0.0 \\
\hline
\end{tabular}




\begin{tabular}{|c|c|c|c|c|c|c|c|c|c|}
\hline \multicolumn{7}{|c|}{$\mathrm{n}$-tridecane } & \multicolumn{3}{|c|}{ iso-dodecane } \\
\hline 80.4 & 4363 & 87.1 & 85.9 & 64.0 & 100.0 & 5872 & 100.0 & 100.0 & 100.0 \\
\hline 40.0 & 2172 & 73.5 & 37.7 & 64.2 & 39.0 & 2288 & 50.0 & 60.0 & 100.0 \\
\hline 20.5 & 1110 & 35.1 & 40.3 & 61.4 & 16.3 & 956 & 11.1 & 50.0 & 10.0 \\
\hline 9.7 & 524 & 34.9 & 29.5 & -4.1 & 6.7 & 391 & 10.0 & 60.0 & N. A. \\
\hline 4.7 & 252 & 19.4 & 21.6 & 55.7 & 2.3 & 136 & 20.0 & 0.0 & 12.5 \\
\hline \multirow[t]{13}{*}{2.5} & 137 & 13.7 & 18.4 & -13.1 & 1.0 & 59 & 0.0 & 10.0 & 60.0 \\
\hline & & & & & & & & D4 & \\
\hline & & & & & 100.0 & 3371 & 100.0 & 100.0 & 100.0 \\
\hline & & & & & 29.9 & 1009 & 100.0 & 100.0 & 28.6 \\
\hline & & & & & 10.1 & 342 & 16.7 & 20.0 & 0.0 \\
\hline & & & & & 3.0 & 100 & 0.0 & 0.0 & 0.0 \\
\hline & & & & & 1.0 & 34 & 0.0 & 0.0 & 0.0 \\
\hline & & & & & & & & D5 & \\
\hline & & & & & 100.0 & 2697 & 70.0 & 22.2 & 80.0 \\
\hline & & & & & 30.1 & 811 & 30.0 & 28.6 & 70.0 \\
\hline & & & & & 10.2 & 274 & 10.0 & 0.0 & 0.0 \\
\hline & & & & & 2.8 & 74 & 0.0 & 10.0 & 0.0 \\
\hline & & & & & 0.9 & 24 & 0.0 & 0.0 & 0.0 \\
\hline
\end{tabular}

